\title{
Border Operator for Generalized Maps
}

\author{
Sylvie Alayrangues ${ }^{1}$, Samuel Peltier ${ }^{1}$, Guillaume Damiand ${ }^{2}$, \\ and Pascal Lienhardt ${ }^{1}$
}

${ }^{1}$ XLIM-SIC, Université de Poitiers, CNRS, Bât. SP2MI, Téléport 2, Bvd Marie et Pierre Curie, BP 3017986962 Futuroscope Chasseneuil Cedex France \{alayrangues, peltier, lienhardt\}@sic.univ-poitiers.fr

${ }^{2}$ Université de Lyon, CNRS, LIRIS, UMR5205, F-69622, France

guillaume.damiand@liris.cnrs.fr

\begin{abstract}
In this paper, we define a border operator for generalized maps, a data structure for representing cellular quasi-manifolds. The interest of this work lies in the optimization of homology computation, by using a model with less cells than models in which cells are regular ones as tetrahedra and cubes. For instance, generalized maps have been used for representing segmented images. We first define a face operator to retrieve the faces of any cell, then deduce the border operator and prove that it satisfies the required property : border of border is void. At last, we study the links between the cellular homology defined from our border operator and the classical simplicial homology.
\end{abstract}

\section{Introduction}

Computing topological properties onto subdivided objects is of interest in different communities of computer science (e.g. computational topology, geometric modeling, discrete geometry, image analysis). Among all topological properties (e.g. Euler characteristic, orientability, connectivity), many works show that homology is a powerful one: it can be computed in the same way in any dimension, it is directly linked with the structure of an object and the homological information can be represented in the object. Different approaches and optimizations have been proposed (e.g. computation of Betti numbers for torsion free objects [5], computation of homology generators [16]).

Most homological results are related to triangulated objects (abstract simplicial sets [15], $\Delta$-complexes [10], semi-simplicial sets [11], simplicial sets [14]). Within these structures, simplices are oriented cells and for any dimension $d>0$, $d+1$ face operators are defined for each $d$-simplex (each face operator maps a $d$-simplex onto a $(d-1)$-simplex; this application is trivial for 0 -simplices). Border operators can then be defined as the alternate sum of the face operators. Homology groups are defined from these border operators. As far as we know, such border operators are defined only for the "regular" structures of simplicial, cubical and simploidal sets [17.

In this paper, we address the problem of computing homology groups for cellular structures (e.g. cell-tuples [4, incidence graphs [18, orders [3]). Such

S. Brlek, C. Reutenauer, and X. Provençal (Eds.): DGCI 2009, LNCS 5810, pp. 300-312, 2009.
(C) Springer-Verlag Berlin Heidelberg 2009 
structures are used in both geometric modeling and image analysis. Any object, represented by any cellular structure, can always be canonically converted onto a simplicial one. Thus, homology of a cellular object can be computed through its associated triangulation, but in this way, the optimization of the cellular structure is lost as the simplicial object is made of more cells than the cellular one. The second drawback is that it is not straightforward to map a simplicial homology generator onto the cellular structure. In order to avoid these drawbacks, we chose to define a border operator for cellular structures.

More precisely, we define a border operator for the structure of generalized maps, which is well-suited for representing cellular quasi-manifolds 1 [13]. It can be noted that there exists several equivalent or derived structures which allow to represent more general cellular complexes or subclasses of quasi-manifolds and this work could be extended for these structures [8]. Moreover structures based on combinatorial maps have already been used in many fields, e.g. image analysis [7], discrete objects modeling [2]. Besides several links between generalized maps and other cellular structures (e.g. cell-tuples [4, orders [3]) have been exhibited and conversion operators between subclasses of such models have also been proposed 112 .

In order to define a border operator for generalized maps, the idea is to match the classical simplicial approach: cells are given an orientation and for each couple $\left(c_{i}, c_{i-1}^{\prime}\right)$, where $c_{i}$ is an $i-$ cell and $c_{i-1}^{\prime}$ an $(i-1)$-cell, the incidence number between $c_{i}$ and $c_{i-1}^{\prime}$ indicates how many times $c_{i}$ is attached to $c_{i-1}^{\prime}$, taking the orientation into account. This operator is actually defined on a restricted subclass of generalized maps which is sufficient to encode most useful subdivisions. We prove that the operator $\partial$ we define is a border operator, i.e. satisfies the property $\partial \partial\left(c_{i}\right)=0$ for any cell $c_{i}, i>0$. Thus, notions of chain, cycle, boundary and homology can be defined. We then study the links between the homology defined from $\partial$ and the simplicial homology (i.e. the homology computed on the associated simplicial structure).

The main advantage of defining a border operator on cellular structures through the explicit characterization of incidence numbers is that the usual methods for homology computation based on Smith normal form reductions of incidence matrices can be used while the optimization of the cellular representation is preserved, and all the optimizations defined for simplicial structures can directly be applied when computing the homology of cellular objects (e.g. computation of generators [16], optimization for incidence matrix reductions into their Smith normal form [6, optimization for sparse incidence matrices [9]). We are currently leading experimental studies to get an accurate estimation of the cellular optimization. In this article, we essentially focus on establishing a theoretical framework. More precisely, we introduce new notions on generalized maps: (un)signed incidence numbers (from section 3 to section 44). These notions are then used to define a new cellular border operator (section 5). All these definitions are based on classical notions related to generalized maps which are

${ }^{1}$ Informally, an $n$-dimensional cellular quasi-manifold is a collection of $n$-cells attached along $(n-1)$-cells such that at most two $n$-cells are incident to a given $(n-1)$-cell. 
carefully recalled (from section 2) in order to ease the understanding and provide a self-contained paper.

In section 2, basic notions of generalized maps and cells are recalled. We also define the notions of compacted cells and face operators (adapted from [8]). This notion is based on an intrinsic property of cellular quasi-manifolds: each cell is a cellular quasi-manifold and can hence be represented with a generalized map of the same dimension. Faces operators associate with each $i$-dimensional cell, all $(i-1)$-cells belonging to its border. The notion of incidence numbers between cells of generalized maps (without taking into account their orientation) is presented in section 3 . The notions of oriented cell and incidence numbers between oriented cells are defined in section 4, In section 5, the border operator of generalized maps is given and we study, in section 6, the links between the cellular homology we have defined and the simplicial homology. Finally, we conclude and give some hints for future works.

\section{Generalized Maps and Corresponding Cell Subdivisions}

A generalized map does not explicitly encode the cells of the associated cellular quasi-manifold [12]. It is constructed on more atomic elements called darts. Each dart is incident to exactly one cell of each dimension. The topological structure of the model is encoded through involutions linking the darts. An $n$-dimensional generalized map is equipped with $(n+1)$ involution 2 . Informally, the involution numbered $k$ relates two darts belonging to the two adjacent $k$-cell 3 incident to the same $i$-cells for all $i$ different from $k$ (Prop. 2 of Def. 11). Moreover cellular quasi-manifolds also have the interesting property to be locally everywhere a cellular quasi-manifold. In particular, the interior of each cell of such a subdivision is a cellular quasi-manifold (Prop. 3 of the Def. 11).

Definition 1. (Generalized map [13]) Let $n \geq 0$. An $n$-dimensional generalized map (or $n G$-map) is $G=\left(D, \alpha_{0}, \ldots, \alpha_{n}\right)$ where:

1. $D$ is a finite set of darts;

2. $\forall i, 0 \leq i \leq n, \alpha_{i}$ is an involution on $D$;

3. $\forall i, j, 0 \leq i<i+2 \leq j \leq n, \alpha_{i} \alpha_{j}$ is an involution.

Subdivided curves are encoded with a set of darts related by two involutions denoted by $\alpha_{0}$ and $\alpha_{1} ; \alpha_{0}$ links darts incident to a same edge and $\alpha_{1}$ relates darts incident to a same vertex (Fig. 1(a)). Similarly, subdivided surfaces are encoded with a set of darts related by three involutions denoted by $\alpha_{0}, \alpha_{1}$ and $\alpha_{2} ; \alpha_{0}$ (resp. $\alpha_{1}, \alpha_{2}$ ) links darts incident to a same edge and face (resp. vertex and face, vertex and edge) (Fig. 1(b)).

Defining the border operator associated with the subdivision requires first to construct the cells of the quasi-manifold from the definition of generalized maps. Actually, each cell is fully and implicitly defined by the set of darts incident to it. Defining a cell usually consists in extracting its corresponding set of darts.

\footnotetext{
${ }^{2}$ An involution $f$ on $S$ is a one-to-one mapping from $S$ to $S$ such that $f=f^{-1}$.

${ }^{3}$ not necessarily different: a cell can be attached to itself.
} 


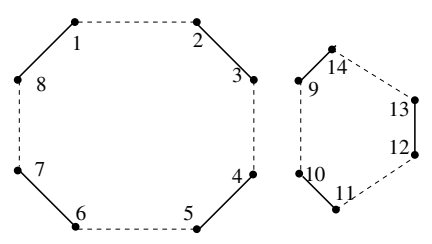

(a) $1 G$-map.

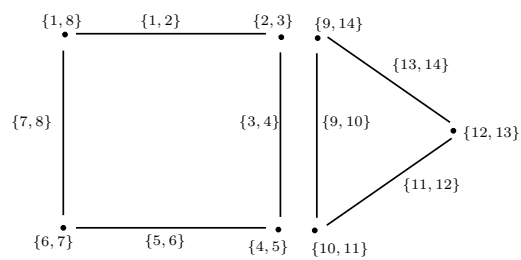

(c) $1 D$-subdivision.

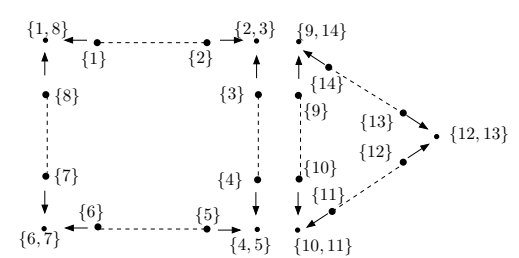

(e) $1 D$ representation based on compacted cells.

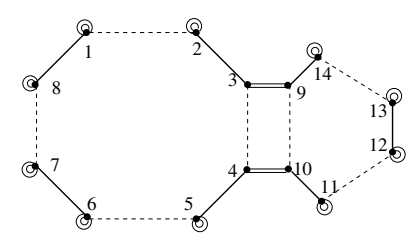

(b) $2 G$-map.

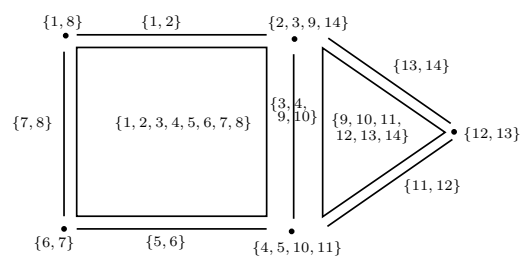

(d) $2 D$-subdivision.

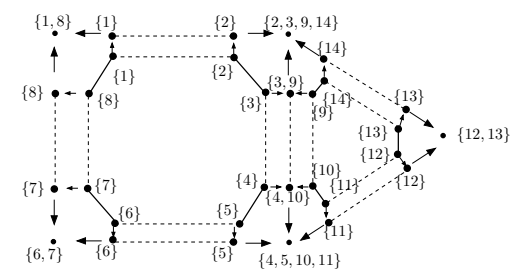

(f) $2 D$ representation based on compacted cells.

Fig. 1. Different representations of a $1 D$-subdivision (left) and a $2 D$-subdivision (right). In the generalized maps depicted on Fig. 1(a) and 1(b) darts are depicted by dots and numbered from 1 , involution $\alpha_{0}$ is represented by dotted lines, $\alpha_{1}$ by plain lines and $\alpha_{2}$ by double lines. On the corresponding subdivisions (Fig. 1(c) and 1(d)), cells are explictly depicted and are associated with their corresponding sets of darts. Face operators are depicted by arrows on the compacted cell decomposition of both subdivisions (Fig. 1(e) and 1(f)].

Definition 2. ( $i$-cell) Let $d$ be a dart and $i \in N=\{0, ., n\}$. The $i$-cell incident to $d$, denoted by $C_{i}(d)$, is the orbit4:

$$
\langle\rangle_{N-\{i\}}(d)=\left\langle\alpha_{0}, \ldots, \alpha_{i-1}, \alpha_{i+1}, \ldots, \alpha_{n}\right\rangle(d)
$$

The $1 D$-subdivision (resp. $2 D$-subdivision) encoded by the $1 G$-map (resp. $2 G$ map) of Fig.1(a) (resp. 1(b) is depicted on Fig 1(c) (resp. 1(d).

This definition of cell is well suited to determine which cells belong to the boundary of another one. More precisely, a cell is in the boundary of another one if they share at least one dart. In the $1 D$ example, vertex $\{1,8\}$ belongs to the boundary of edges $\{1,2\}$ and $\{7,8\}$ and in the $2 D$ example, vertex $\{2,3,9,14\}$

\footnotetext{
${ }^{4}$ Let $\left\{\Pi_{0}, \ldots, \Pi_{n}\right\}$ be a set of permutations on $D$. The orbit of an element $d$ relatively to this set of permutations is $\left\langle\Pi_{0}, \ldots, \Pi_{n}\right\rangle(b)=\left\{\Phi(b), \Phi \in\left\langle\Pi_{0}, \ldots, \Pi_{n}\right\rangle\right\}$, where $\left\langle\Pi_{0}, \ldots, \Pi_{n}\right\rangle$ denotes the group of permutations generated by $\left\{\Pi_{0}, \ldots, \Pi_{n}\right\}$.
} 
belongs to the boundary of edges $\{1,2\},\{3,4,9,10\}$ and $\{13,14\}$. However, this notion of boundary is not rich enough to deduce a well-defined border operator. This definition does not accurately describe multi-incidences (e.g. in the subdivision depicted on Fig. 2 representing a cylinder, vertex $\{1,6,7,8\}$ is twice incident to edge $\{7,8\}$, and edge $\{1,2,5,6\}$ is twice incident to face $\{1,2,3,4,5,6,7,8\}$ ). It can also be noted that the two minimal subdivisions of a sphere and a projective plane cannot be distinguished using this notion of boundary [1].

In order to have a representation which is both able to explicitly represent cells and compliant with an accurate face operator, we define compacted cells. This notion is based on the fact that the interior of each cell is a quasi-manifold and can hence be represented by a generalized map of the same dimension. The set of darts of a generalized map representing $i$-cells is isomorphic to the set of orbits $\left\langle\alpha_{i+1}, \ldots, \alpha_{n}\right\rangle$ of the generalized map encoding the whole subdivision. Involutions $\alpha_{0}^{i}, \ldots, \alpha_{i-1}^{i}$ are straightforwardly deduced from $\alpha_{0}, \ldots, \alpha_{i-1}$.

Definition 3. (compacted $i$-cell) Let $i \in\{0, ., n\}$.

Let $B^{i}=\left\{\left\langle\alpha_{i+1}, \ldots, \alpha_{n}\right\rangle(d), d \in D\right\}$. Involutions $\alpha_{k}^{i}, k \in\{0, \ldots, i-1\}$ are defined on $B^{i}$ by:

$$
\forall d \in D,\left(\left\langle\alpha_{i+1}, \ldots, \alpha_{n}\right\rangle(d)\right) \alpha_{k}^{i}=\left\langle\alpha_{i+1}, \ldots, \alpha_{n}\right\rangle\left(\left(d \alpha_{k}\right)\right)
$$

The compacted $i$-cell incident to $d, c_{i}(d)$, is the set of elements of $B^{i}$ connected to $\left\langle\alpha_{i+1}, \ldots, \alpha_{n}\right\rangle(d)$ by a composition of involutions $\alpha_{k}^{i}, k \in\{0, \ldots, i-1\}$.

As involutions $\alpha_{k}^{i}$ are defined on the sets $B^{i}$ for $k<i$, property 3 of Definition 1 grants that each $B^{i}$ equipped with these involutions is a generalized map.

The notion of compacted cells is illustrated on Fig. 1(e) (1D-subdivision) and $1(\mathrm{f})(2 D$-subdivision). For instance, edge $\{3,4,9,10\}$ of Fig. $1(\mathrm{~d})$ is represented by the $\alpha_{0}^{1}$-connected set $\left\{\left\langle\alpha_{2}\right\rangle(3)=\{3,9\},\left\langle\alpha_{2}\right\rangle(4)=\{4,10\}\right\}$ (Fig. 1(f) $)$.

A set of face operators describes the links between cells and cells of their boundaries. The $i^{t h}$ face operator formalizes an incidence relation between a compacted $i$-cell and a compacted $(i-1)$-cell incident to it. More precisely, it associates darts of $B^{i}$ and $B^{i-1}$ in such a way that it is coherent upon the cells.

Definition 4. (face operator) Let $i \in\{1, ., n\}$.

The face operator $\partial_{i}$, is a mapping from $B^{i}$ to $B^{i-1}$ defined by:

$$
\left\langle\alpha_{i+1}, \ldots, \alpha_{n}\right\rangle(d) \stackrel{\partial_{i}}{\longrightarrow}\left\langle\alpha_{i}, \ldots, \alpha_{n}\right\rangle(d)
$$

It can be proven that $\partial_{i} \alpha_{k}^{i-1}=\alpha_{k}^{i} \partial_{i}$ for all $k \in[0, i-2]$ (Prop. 3 of Def. 11) . $\partial_{i}^{-1}$ denotes the preimage of $\partial_{i}$. For instance, on Fig. 1(e), the image of the darts \langle\rangle$(2)=\{2\}$ and \langle\rangle$(3)=\{3\}$ (belonging to $B^{1}$ ) by the face operator, $\partial_{1}$, is the set $\{2,3\}=\left\langle\alpha_{1}\right\rangle(2)=\left\langle\alpha_{1}\right\rangle(3)$ (belonging to $\left.B^{0}\right)$. On Fig. 1(f), the image by $\partial_{1}$ of the singleton $\left\langle\alpha_{2}\right\rangle(1)=\{1\}$ belonging to $B^{1}$ is the set $\{1,8\}=\left\langle\alpha_{1}, \alpha_{2}\right\rangle(1)$ belonging to $B^{0}$. The image of the darts of $B^{2},\langle\rangle(2)=\{2\}$ and \langle\rangle$(3)=\{3\}$ by $\partial_{2}$ are respectively the sets $\{2\}=\left\langle\alpha_{2}\right\rangle(2)$ and $\{3,9\}=\left\langle\alpha_{2}\right\rangle(3)$. 


\section{Unsigned Incidence Numbers}

The unsigned incidence number is defined for any pair of cells whose dimensions differ by exactly 1 . It counts how many times the cell of lesser dimension is present in the boundary of the cell of greater dimension. This number can be obtained from the face operator. Given a dart of the cell of lesser dimension, we only need to compare its preimage under the face operator and the cell of greater dimension. The unsigned incidence number is equal to the number of elements of the preimage whose darts also belong to the cell. Otherwise stated, for two cells, $C_{i}(d)$ and $C_{i-1}\left(d^{\prime}\right)$, the unsigned incidence number is equal to the number of distinct orbits $\left\langle\alpha_{i+1}, \ldots, \alpha_{n}\right\rangle$ contained in $\left\langle\alpha_{i}, \ldots, \alpha_{n}\right\rangle\left(d^{\prime}\right)$ whose darts are also included in $C_{i}(d)=\langle\rangle_{N-\{i\}}(d)$.

Definition 5. (unsigned incidence number) Let $i \in\{1, ., n\}$. Let $C_{i}(d)$ and $C_{i-1}\left(d^{\prime}\right)$ be two cells of the subdivision encoded by $G$. The incidence number, $\left(C_{i}(d): C_{i-1}\left(d^{\prime}\right)\right)$, is:

$$
\begin{gathered}
\operatorname{card}\left(\left\{\left\langle\alpha_{i+1}, \ldots, \alpha_{n}\right\rangle\left(d^{\prime \prime}\right) \in \partial_{i}^{-1}\left(\left\langle\alpha_{i}, \ldots, \alpha_{n}\right\rangle\left(d^{\prime}\right)\right)\right.\right. \\
\text { s.t. } \left.\left.\left\langle\alpha_{i+1}, \ldots, \alpha_{n}\right\rangle\left(d^{\prime \prime}\right) \subseteq C_{i}(d)\right\}\right)
\end{gathered}
$$

Property 1. $\left(C_{i}(d): C_{i-1}\left(d^{\prime}\right)\right)$ does not depend on the chosen darts. This incidence number is hence denoted by $\left(C_{i}: C_{i-1}\right)$.

The proof is a straightforward consequence of property 3 of Definition 1

For instance, let us compute the incidence number between the edge incident to 14 and the face incident to 1 on Fig. 1(f). The edge is actually represented by the set $\left\{\left\langle\alpha_{2}\right\rangle(14)=\{14\},\left\langle\alpha_{2}\right\rangle(13)=\{13\}\right\}$ connected by the involution $\alpha_{0}^{1}$ and the face by the set $\{\{1\},\{2\},\{3\},\{4\},\{5\},\{6\},\{7\},\{8\}\}$ connected by $\alpha_{0}^{2}, \alpha_{1}^{2}$. The preimage of the singleton $\left\langle\alpha_{2}\right\rangle(14)=\{14\}$ (belonging to $B^{1}$ ) is the singleton \langle\rangle$(14)=\{14\}$ (belonging to $B^{2}$ ) which is not included into the face incident to dart 1 . The unsigned incidence number of both cells is hence 0 . Let us consider the edge incident to dart 9 relatively to the same face. This edge is the set $\left\{\left\langle\alpha_{2}\right\rangle(9),\left\langle\alpha_{2}\right\rangle(10)\right\}=\{\{3,9\},\{4,10\}\}$ connected via $\alpha_{0}^{1}$. The preimage of $\left\langle\alpha_{2}\right\rangle(9)$ is the set made of darts $\{3\}$ and $\{9\}$. Its intersection with the face $\{\{1\},\{2\},\{3\},\{4\},\{5\},\{6\},\{7\},\{8\}\}$ contains exactly one element: $\{3\}$. The incidence number between both cells is thus equal to 1 .

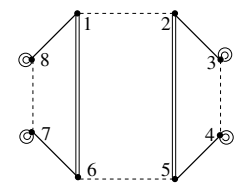

(a) $2 G$-map

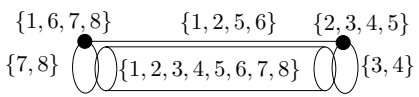

(b) $2 D$-subdivision

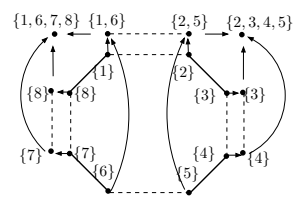

(c) Compacted cells representation

Fig. 2. Representation of a cylinder 
Let us consider, on Fig. 2, the vertex incident to dart 1, $\left\{\left\langle\alpha_{1}, \alpha_{2}\right\rangle(1)=\right.$ $\{1,6,7,8\}\}$, and the edge incident to the same dart, $\left\{\left\langle\alpha_{2}\right\rangle(1)=\{1,6\},\left\langle\alpha_{2}\right\rangle(2)=\right.$ $\{2,5\}\}$. The preimage of $\{1,6,7,8\}$ is the set $\{\{1,6\},\{7\},\{8\}\}$. Only one subset of the preimage is included in the edge, the incidence number is thus equal to 1 . The edge incident to dart 8 is $\left\{\left\langle\alpha_{2}\right\rangle(8)=\{8\},\left\langle\alpha_{2}\right\rangle(7)=\{7\}\right\}$ connected by $\alpha_{0}^{1}$. Two subsets of the preimage of the vertex incident to dart 1 , $\{\{1,6,7,8\}\}$, are included in the edge incident to 8 . The corresponding incidence number is hence 2 . This subdivision contains a single face \langle\rangle$(1)=$ $\{\{1\},\{2\},\{3\},\{4\},\{5\},\{6\},\{7\},\{8\}\}$. The preimage of $\{8\}$, used to compute the incidence number between the edge incident to dart 8 and the face of the subdivision, is the singleton $\{8\}$. It is included in the face, the incidence number associated to this pair of cells is hence 1 . The preimage of $\alpha_{2}(1)=\{1,6\}$, corresponding to the edge incident to dart 1 , is the set $\{\{1\},\{6\}\}$. Both elements belong to the face, the corresponding incidence number is thus 2 . Note that considering the other element of the same edge, $\alpha_{2}(2)=\{2,5\}$ instead of dart $\{1,6\}$ leads to the same result. Its preimage is the set $\{\{2\},\{5\}\}$ whose both elements also belong to the face: this is consistent with Property 1 .

The definitions and properties presented in the beginning of this section hold for any kind of generalized maps, e.g. maps whose involutions may have fixed point5. Such generalized maps are able to encode cellular quasi-manifolds whose cells may have incomplete boundaries [13, i.e. the boundary of an $i$-cell is not equal to the union of incident $j$-cells, $j<i$. In the following, we intend to define a border operator acting on cells and need hence the cells of the subdivision to have a well-defined boundary. We restrict thus the remaining of the study to the subclass of generalized maps defined as follows:

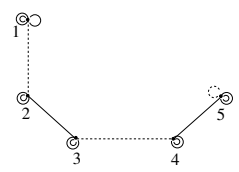

(a) $2 G$-map that does not fulfill Property 1 of Definition $6 \alpha_{0}$ and $\alpha_{1}$ have fixed points (resp. dart 5 and 1)

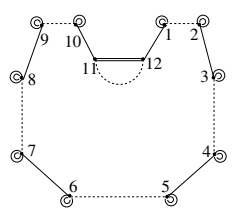

(c) $2 G$-map that does not fulfill Property 2 of Definition 6

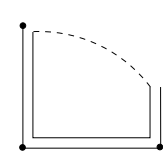

(b) An edge and a face of the corresponding subdivision do not have a complete boundary

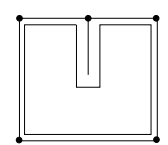

(d) The corresponding subdivision has an inner edge with incomplete boundary

Fig. 3. 2G-maps containing cells with incomplete boundary

\footnotetext{
${ }^{5} b$ is a fixed point for some involution $\alpha \Leftrightarrow b \alpha=b$.
} 
Definition 6. An $n G$-map, $G$, is said to have cells with complete boundary if:

1. $\alpha_{i}$ is without fixed point, $0 \leq i \leq n-1$

2. $G$ is without self-bending: $\forall d \in D, \forall i \in\{0, \ldots, n\},\left\langle\alpha_{i+1}, \ldots, \alpha_{n}\right\rangle(d) \cap\left\langle\alpha_{0}, \ldots, \alpha_{i-1}\right\rangle(d)=\{d\}$.

Counter-examples are displayed on Fig. 3.

Property 2. In such a generalized map, involutions defined on each compacted $i$-cell, $\left(\alpha_{0}^{i}, \ldots, \alpha_{i-1}^{i}\right)$, are without fixed points.

Note that this restriction to generalized maps without self-bending does not forbid subdivisions to have some multi-incidence (Fig. 2).

\section{Cell Orientation and Signed Incidence Numbers}

This section aims at characterizing more precisely the incidence between two cells not only by studying how many times a cell is incident to another one but also how these cells are connected, i.e. along which "directions". For instance, a cell which is incident twice to another one may be once incident along one "direction" and once along the opposite "direction". Such considerations are accurate only if it is possible to define the notion of "directions", that is to give an orientation to each cell of the subdivision. We define thus the notions of orientable and oriented (compacted) cells. As cells of a subdivision can be encoded by generalized maps, we recall first the notion of orientability for generalized maps.

Definition 7. (orientable generalized map) A generalized map is orientable if its set of darts can be partitioned into two classes such that if $d$ belongs to one class, $d \alpha_{i}$ belongs to the other for all $i \in\{0, \ldots, n\}$ when $d \alpha_{i} \neq d$.

Definition 8. (oriented generalized map) Let $G$ be an orientable $n G$-map. The oriented generalized map, $\vec{G}$, is $G$ in which one of the two classes is chosen 6 . In $\vec{G}$, the chosen class is denoted by $\vec{G}^{+}$, the other one by $\vec{G}^{-}$.

The inverse orientation is denoted by $-\vec{G}$.

A single dart d may be chosen to represent the class $\vec{G}$, the oriented generalized map is hence denoted by $\vec{G}(d)$

In the remaining of the paper, we only deal with orientable or not orientable cellular subdivisions, whose cells have complete boundaries and are orientable (Fig. 5(a) and 5(b) . For instance, the Klein bottle is not orientable but all its (compacted) cells are (Fig. 4(b)). It can be easily proved that the compacted cells of such subdivisions are orientable. So an oriented compacted cell is denoted either by $\vec{c}_{i}$ or by $-\vec{c}_{i}$. In practice, each dart can be given a mark, + or - , for each dimension $i$. This mark points out the orientation class of the corresponding compacted $i$-cell to which the dart belongs. To obtain a consistent marking, all 


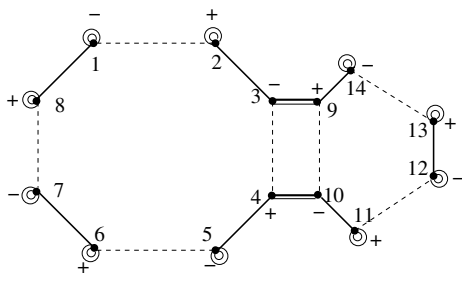

(a) Orientable $2 G$-map with an orientation.

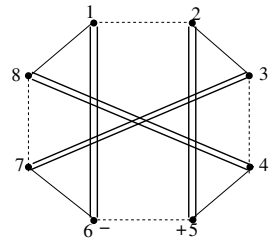

(b) Non orientable 2G-map encoding a Klein bottle.

Fig. 4. Orientability of $2 G$-maps

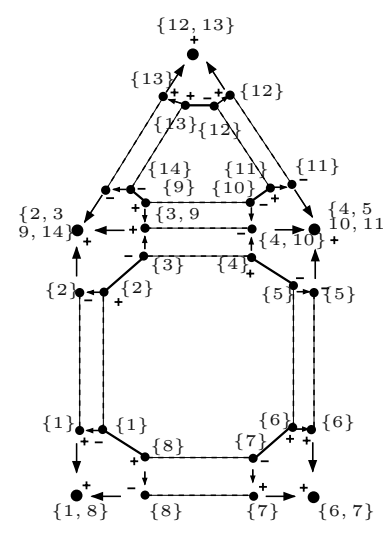

(a) Representation of the subdivision with oriented compacted cells.

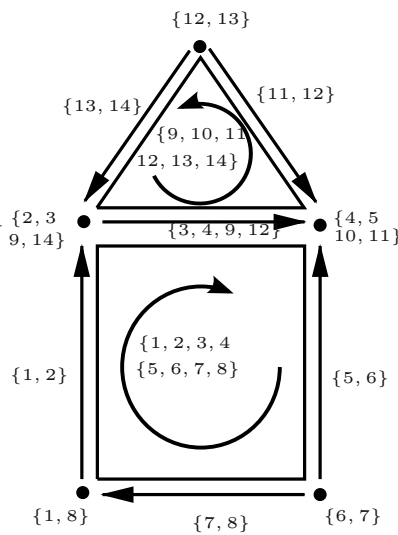

(b) $2 D$-subdivision where each cell is oriented from + to - .

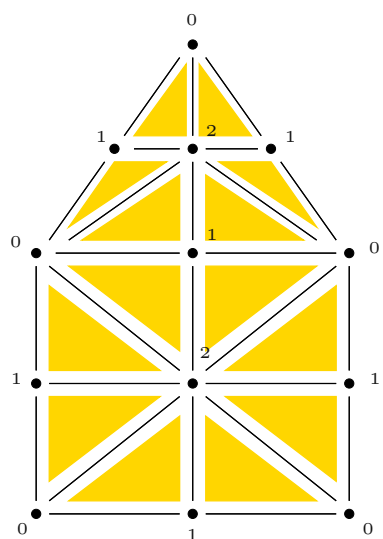

(c) Numbered simplicial object.

darts contained in an $\left\langle\alpha_{i+1}, \ldots, \alpha_{n}\right\rangle$-orbit must have the same mark for the $i^{\text {th }}$ dimension (Fig. 5(a)).

Defining the signed incidence number between an $i$-cell and an $(i-1)$-cell is quite similar to defining their associated unsigned incidence number. The preimage under $\partial_{i}$ of some dart $\left\langle\alpha_{i+1}, \ldots, \alpha_{n}\right\rangle(d)$ of a compacted $(i-1)$-cell, $c_{i-1}(d)$, is examined. The elements of this preimage that are included in the $i$-cell, $c_{i}=c_{i}\left(d^{\prime}\right)$ are counted but a coefficient +1 or -1 is used to take their relative orientation into account.

Definition 9. (signed incidence number) Let $i \in\{1, \ldots, n\}$. Let $\overrightarrow{c_{i-1}}$ and $\overrightarrow{c_{i}}$ be two compacted oriented cells of the subdivision, and $\overrightarrow{C_{i-1}}, \overrightarrow{C_{i}}$ the associated oriented cells. Let $d$ be a dart incident to $C_{i-1}$. The signed incidence number of $\left(\overrightarrow{C_{i}}: \overrightarrow{C_{i-1}}\right)=\left(\overrightarrow{c_{i}}: \overrightarrow{c_{i-1}}\right)$ is:

${ }^{6}$ In practice, each dart can be given a mark, e.g. + or - if it belongs to $\vec{G}^{+}$or to $\vec{G}^{-}$(Fig. 4(a)]. 


$$
\sum_{\substack{\left\langle\alpha_{i+1}, \ldots, \alpha_{n}\right\rangle\left(d^{\prime}\right) \in \partial_{i}^{-1}\left(\left\langle\alpha_{i}, \ldots, \alpha_{n}\right\rangle(d)\right) \\ \text { s.t. }\left\langle\alpha_{i+1}, \ldots, \alpha_{n}\right\rangle\left(d^{\prime}\right) \subseteq C_{i}}} \operatorname{sign}\left(\overrightarrow{c_{i}}\left(d^{\prime}\right), \overrightarrow{c_{i-1}}(d)\right)
$$

$$
\begin{aligned}
\operatorname{sign}\left(\overrightarrow{c_{i}}\left(d^{\prime}\right), \overrightarrow{c_{i-1}}(d)\right)= & +1 \text { if } d \in \overrightarrow{c_{i-1}}+\text { and } d^{\prime} \in \overrightarrow{c_{i}}+\text { or } d \in \overrightarrow{c_{i-1}}-\text { and } d^{\prime} \in \overrightarrow{c_{i}}- \\
& -1 \text { otherwise. }
\end{aligned}
$$

Property 3. The definition of the signed incidence number is consistent.

The proof is similar to the proof of Property 1 for unsigned incidence number but both possible orientations of $c_{i-1}$ and $c_{i}$ have to be taken into account.

\section{Border Operator}

Having defined the notion of signed incidence number between any pair $\left(\vec{C}_{i}, \vec{C}_{i-1}\right)$, we can define the border of an $i$-cell by extending this notion linearly onto all $(i-1)$-cells belonging to the boundary of an $i$-cell.

Definition 10. (Border Operator) Let $\mathfrak{C}_{i-1}$ be the set of $(i-1)$-cells. Let $\vec{C}_{i}$ be an oriented $i$-cell, the border of $\vec{C}_{i}$ is:

$$
\vec{C}_{i} \partial^{C}=\sum_{\vec{C}_{i-1}^{j} \in \mathfrak{C}_{i-1}}\left(\vec{C}_{i}: \vec{C}_{i-1}^{j}\right) \vec{C}_{i-1}^{j}
$$

This definition can be extended for any integer weighted sum of cells, classically called chains of cells. Moreover this notion of border is compliant with the definition of an homology because of property 4 ;

Property 4. $\partial^{C} \partial^{C}=0$

The proof is based on the following idea. Let $d$ be a dart incident to a given oriented compacted $i$-cell, $\overrightarrow{c_{i}}$ and let $\overrightarrow{c_{i-2}}$ be the oriented compacted $(i-2)$ cell incident to $d$, such that $d$ belongs to $\overrightarrow{c_{i}}+$ and $\overrightarrow{c_{i-2}}+$. The preimage of $\left\langle\alpha_{i-1}, \ldots, \alpha_{n}\right\rangle(d)$ under $\partial_{i-1}$ contains $\left\langle\alpha_{i}, \ldots, \alpha_{n}\right\rangle(d)$, whose preimage under $\partial_{i}$ contains $\left\langle\alpha_{i+1}, \ldots, \alpha_{n}\right\rangle(d)$. This orbit is obviously included in the $i$-cell. Note that $d \alpha_{i-1}$ belongs to $\overrightarrow{c_{i}}-$. The image of $\left\langle\alpha_{i+1}, \ldots, \alpha_{n}\right\rangle\left(d \alpha_{i-1}\right)$ under $\partial_{i},\left\langle\alpha_{i}, \ldots, \alpha_{n}\right\rangle\left(d \alpha_{i-1}\right)$ belongs to a compacted $(i-1)$-cell, and its image under $\partial_{i-1}$ is still $\overrightarrow{c_{i-2}(d)}=\overrightarrow{c_{i-2}} \cdot \overrightarrow{c_{i-2}}$ is hence present in $\left(\overrightarrow{c_{i}}\right) \partial^{C} \partial^{C}$, once with a sign for $\left\langle\alpha_{i+1}, \ldots, \alpha_{n}\right\rangle(d)$ and once with the opposite sign for $\left\langle\alpha_{i+1}, \ldots, \alpha_{n}\right\rangle\left(d \alpha_{i-1}\right)$. This property is true whatever the orientations of incidente $(i-1)$-cells are. For instance, on Fig. 5(a) consider dart 2 and the 0 -cell and the 2 -cell to which it is incident. In the preimage of the vertex incident to 2 , two elements are "incident" to the 2-cell with different orientations. Both elements are related by $\alpha_{1}^{1}$.

The incidence matrix used to compute the homology groups of a generalized map, belonging to the subclass considered here, is straightforwardly obtained via the signed incidence number. 


\section{Towards the Equivalence between Cellular and Simplicial Homology}

In this section, the relation between simplicial homology and the cellular homology we have defined from the cellular boundary operator $\partial^{c}$ is studied. It is always possible to associate with any $n G$-map, a simplicial object such that:

- each dart corresponds to a $n$-simplex numbered $\{0, \cdots, n\}$,

- two $n$-simplices obtained from two darts linked by $\alpha_{i}$ share a $(n-1)$-face numbered $\{0, \cdots, \widehat{i}, \cdots, n\}$.

For example in Fig. 5(c), each dart corresponds to a triangle numbered $\{0,1,2\}$. The two triangles corresponding to the darts 4 and 5 are linked by $\alpha_{1}$ and incident to an edge numbered $\{0,2\}$.

Thus, a compacted $i$-cell is made of one vertex $v$ numbered $i$, and a collection of $j$-simplices, $1 \leq j \leq i$, incident to $v$, numbered by integers lowers than $i$ (denoted by $\{\ldots, i\})$.

Given a dart $d$ and its associated oriented compacted cell $\overrightarrow{c_{i}}(d)$, we define $\overrightarrow{c_{i}}(d) \tau$ as the sum of the $i$-dimensional simplices of $c_{i}(d)$, taken positivey if the corresponding dart has the orientation of $d$, and negatively otherwise. The operator $\tau$ can directly be extended by linearity to sum of compacted cells.

If we denote the cellular boundary operator $\partial^{c}$ and the simplicial one by $\partial^{s}$, we can show for any compacted $i$-cell $c_{i}$, that $c_{i} \partial^{c} \tau=c \tau \partial^{s}$. This is due to the fact that the interior of any compacted cell is a quasi manifold, so only one $(i-1)$-simplex $\sigma$ belongs to the boundary of two $i$-simplices linked by $\alpha_{j}^{i}$. Since they have inverse orientation, $\sigma$ does not appear in the boundary of $c_{i}$.

We can thus deduce that for any cellular cycle $z$ (resp. boundary), $z \tau$ is a simplicial cycle (resp. boundary): for instance, let $z$ be a cellular cycle, then $z \partial=0 \Rightarrow z \partial^{c} \tau=0 \Rightarrow z \tau \partial^{s}=0 \Rightarrow z \tau$ is a cycle.

In order to prove the equivalence between cellular and simplicial homology, the converse has to be proved: for any simplicial cycle (resp. boundary) $z$, then there exists a cellular cycle (resp. boundary) $z^{c}$ such that $z$ and $z^{c} \tau$ are homologuous.

It can easily be shown that, if a simplicial $i$-cycle (resp. $i$-boundary) $z$ is exclusively made of $i$-simplices associated with darts of compacted $i$-cells (i.e. simplices numbered $\{0, \cdots, i\}$ ), then there exists a cellular cycle (resp. boundary) $z^{c}$ such that $z^{c} \tau=z$ (this is due to the fact that compacted $i$-cells are quasi-manifolds, so if $z$ is exclusively made of $i$-simplices numbered $\{0, \cdots, i\}$, then all the simplices corresponding to a cell must appear in $z$, or else there is no chance that $z$ is a cycle). When a simplicial $i$-cycle contains some $i$-simplices $i n$ ternal to a cell triangulation $c \tau$ (i.e. $\operatorname{dim}(c)>i$ and these simplices are numbered $\{\cdots, \operatorname{dim}(c)\})$, then we need to show that we can replace this chain of simplices by an homologuous chain such that each simplex is numbered $\{\cdots, \operatorname{dim}(c)-1\}$. So we could always replace a part of $z$ by an homologuous chain, so that $z$ remains a cycle and by recursion, we finally would obtain a simplicial cycle $z^{\prime}$ homologuous to $z$ and exclusively made of simplices numbered $\{0, \cdots, i\}$.

\footnotetext{
${ }^{7} z$ is a cycle if $z \partial=0 ; z$ is a boundary, if $c$ exists, such that $c \partial=z$
} 
Thus, each cellular cycle (resp. boundary) could be associated with a simplicial cycle (resp. boundary) and reciprocally. Thus there will be an isomorphism between cellular and simplicial homology groups.

\section{Conclusion}

To conclude, we have defined a border operator for generalized maps having orientable cells with complete boundaries. It has been shown that this border operator defines a cellular homology and the proof of its equivalence with the simplicial homology is actually under study. Techniques of homology computation and their optimizations classically defined on simplicial structures can now directly be used for cellular structures.

For future works, we are studying some possible optimizations for particular cases of cellular structures (e.g. orientable generalized maps) and the extension of the border operator definition to more general cellular structures (complex maps, incidence graphs...).

In order to efficiently compute the homology of any cellular structure, another approach is needed: in the general case, cells may not be orientable. A track that should be followed is that any cellular structure can be interpreted as a simplicial one (structured into cells), and built as a succession of specific operations. So, we are working on the adaptation of incremental approaches defined for the computation of simplicial structures homology [19].

\section{References}

1. Alayrangues, S., Daragon, X., Lachaud, J.-O., Lienhardt, P.: Equivalence between closed connected n-g-maps without multi-incidence and n-surfaces. J. Math. Imaging Vis. 32(1), 1-22 (2008)

2. Andres, E., Breton, R., Lienhardt, P.: Spamod: design of a spatial modeler. In: Bertrand, G., Imiya, A., Klette, R. (eds.) Digital and Image Geometry. LNCS, vol. 2243, pp. 90-107. Springer, Heidelberg (2002)

3. Bertrand, G.: New notions for discrete topology. In: Bertrand, G., Couprie, M., Perroton, L. (eds.) DGCI 1999. LNCS, vol. 1568, pp. 218-228. Springer, Heidelberg (1999)

4. Brisson, E.: Representing geometric structures in d dimensions: Topology and order. Discrete \& Computational Geometry 9, 387-426 (1993)

5. Delfinado, C.J.A., Edelsbrunner, H.: An incremental algorithm for betti numbers of simplicial complexes on the 3-sphere. Comput. Aided Geom. Design 12(7), 771-784 (1995)

6. Dumas, J.-G., Heckenbach, F., Saunders, B.D.: Computing simplicial homology based on efficient smith normal form algorithms. In: Algebra, Geometry, and Software Systems, pp. 177-206 (2003)

7. Dupas, A., Damiand, G.: First results for 3D image segmentation with topological map. In: Coeurjolly, D., Sivignon, I., Tougne, L., Dupont, F. (eds.) DGCI 2008. LNCS, vol. 4992, pp. 507-518. Springer, Heidelberg (2008)

8. Elter, H., Lienhardt, P.: Cellular complexes as structured semi-simplicial sets. International Journal of Shape Modeling 1(2), 191-217 (1995) 
9. Giesbrecht, M.: Probabilistic computation of the smith normal form of a sparse integer matrix. In: Cohen, H. (ed.) ANTS 1996. LNCS, vol. 1122, pp. 173-186. Springer, Heidelberg (1996)

10. Hatcher, A.: Algebraic Topology. Cambridge University Press, Cambridge (2002)

11. Hu, S.T.: On the realizability of homotopy groups and their operations. Pacific J. of Math. 1(583-602) (1951)

12. Lienhardt, P.: Topological models for boundary representation: a comparison with n-dimensional generalized maps. Comput. Aided Design 23(1), 59-82 (1991)

13. Lienhardt, P.: N-dimensional generalized combinatorial maps and cellular quasimanifolds. Int. J. on Comput. Geom. \& App. 4(3), 275-324 (1994)

14. May, J.P.: Simplicial Objects in Algebraic Topology. Van Nostrand (1967)

15. Munkres, J.R.: Elements of algebraic topology. Perseus Books, Cambridge (1984)

16. Peltier, S., Alayrangues, S., Fuchs, L., Lachaud, J.-O.: Computation of homology groups and generators. Comput. \& Graph. 30, 62-69 (2006)

17. Peltier, S., Fuchs, L., Lienhardt, P.: Simploidals sets: Definitions, operations and comparison with simplicial sets. Discrete App. Math. 157, 542-557 (2009)

18. Rossignac, J., O'Connor, M.: A dimension-independant model for pointsets with internal structures and incomplete boundaries. Geometric modeling for Product Engineering, 145-180 (1989)

19. Sergeraert, F.: Constructive algebraic topology. SIGSAM Bulletin 33(3), 13-13 (1999) 